Homology, Homotopy and Applications, vol.11(2), 2009, pp.49-54

\title{
ON COMPLEXES OF FINITE COMPLETE INTERSECTION DIMENSION
}

\author{
PETTER ANDREAS BERGH
}

(communicated by J.P.C. Greenlees)

\begin{abstract}
We study complexes of finite complete intersection dimension in the derived category of a local ring. Given such a complex of complexity $c$, we prove that the thick subcategory it generates contains complexes of all possible complexities at most $c$. In particular, we show that such a complex is virtually small, answering a question raised by Dwyer, Greenlees and Iyengar.
\end{abstract}

\section{Introduction}

Given a homologically finite complex over a local ring, the complexity of the complex measures the polynomial rate of growth in its minimal free resolution. The rings over which all such complexes have finite complexity are precisely the local complete intersections. If a complex has finite complete intersection dimension (see Section 3), then it behaves homologically as a complex over a complete intersection. In particular, complexes of finite complete intersection dimension have finite complexity.

The main result of this paper concerns the thick subcategory thick ${ }_{D(A)}(M)$ generated by a nonzero homologically finite complex $M$ of finite complete intersection dimension over a local ring $A$. We show that if the complexity of $M$ is $c$, then thick $_{D(A)}(M)$ contains a nonzero complex of complexity $t$ for every $0 \leqslant t \leqslant c$. In particular, we show that the thick subcategory generated by $M$ contains a nonzero perfect complex, that is, a complex of finite projective dimension, thus answering a question raised in [8, Remarks 9.6].

\section{Acknowledgements}

I would like to thank Srikanth Iyengar and the referee for valuable comments on this paper.

\section{Notation and terminology}

Let $(A, \mathfrak{m}, k)$ be a local (meaning commutative Noetherian local) ring, and denote by $D(A)$ the derived category of (not necessarily finitely generated) $A$-modules. A

The author was supported by NFR Storforsk grant no. 167130 .

Received May 19, 2009, revised June 23, 2009; published on July 28, 2009.

2000 Mathematics Subject Classification: 13D25, 18E30, 18G10.

Key words and phrases: finite complete intersection dimension, complexity, virtually small complexes.

This article is available at http://intlpress.com/HHA/v11/n2/a4

Copyright (c) 2009, International Press. Permission to copy for private use granted. 
complex

$$
M: \cdots \rightarrow M_{n+1} \rightarrow M_{n} \rightarrow M_{n-1} \rightarrow \cdots
$$

in $D(A)$ is bounded below if $M_{n}=0$ for $n \ll 0$, and bounded above if $M_{n}=0$ for $n \gg 0$. The complex is bounded if it is both bounded below and bounded above, and finite if it is bounded and degreewise finitely generated. The homology of $M$, denoted $\mathrm{H}(M)$, is the complex with $\mathrm{H}(M)_{n}=\mathrm{H}_{n}(M)$, and with trivial differentials. When $\mathrm{H}(M)$ is finite, then $M$ is said to be homologically finite.

As shown, for example, in [10], when the complex $M$ is homologically finite, it has a minimal free resolution. Thus, there exists a quasi-isomorphism $F \simeq M$, where $F$ is a bounded below complex

$$
\cdots \rightarrow F_{n+1} \stackrel{d_{n+1}}{\longrightarrow} F_{n} \stackrel{d_{n}}{\longrightarrow} F_{n-1} \rightarrow \cdots
$$

of finitely generated free modules, and where $\operatorname{Im} d_{n} \subseteq \mathfrak{m} F_{n-1}$. This resolution is unique up to isomorphism, and so for each integer $n$ the rank of the free module $F_{n}$ is a well-defined invariant of $M$. This is the $n$th Betti number $\beta_{n}(M)$ of $M$, and the corresponding generating function $\sum_{n=0}^{\infty} \beta_{n}(M) t^{n}$ is the Poincaré series $P(M, t)$ of $M$. The complexity of $M$, denoted cx $M$, is defined as

$$
\operatorname{cx} M \stackrel{\text { def }}{=} \inf \left\{t \in \mathbb{N} \cup\{0\} \mid \exists a \in \mathbb{R} \text { such that } \beta_{n}(M) \leqslant a n^{t-1} \text { for } n \gg 0\right\} .
$$

This notion was introduced for modules over local rings by Avramov in [1] and [2], and adopted to complexes by Sather-Wagstaff in [12]. The complexity of a homologically finite complex is not necessarily finite. In fact, it follows from a theorem of Gulliksen (cf. [9]) that finiteness of the complexities of all homologically finite complexes in $D(A)$ is equivalent to $A$ being a complete intersection ring. Gulliksen stated this result in terms of finitely generated modules, but the proof carries over to complexes. Note that a complex has complexity zero if and only if its Betti numbers eventually vanish. Thus, the complexes of complexity zero are precisely those of finite projective dimension, that is, the perfect complexes.

Suppose that our complex $M$ is homologically finite, with a minimal free resolution $F$. Given a complex $N \in D(A)$, the complex $\operatorname{Hom}_{A}(F, N)$ is denoted by $\mathbf{R} \operatorname{Hom}_{A}(M, N)$. Up to quasi-isomorphism, this complex is well defined, hence so is the cohomology group

$$
\operatorname{Ext}_{A}^{n}(M, N) \stackrel{\text { def }}{=} \mathrm{H}_{-n}\left(\mathbf{R} \operatorname{Hom}_{A}(M, N)\right)
$$

for every integer $n$. When $N=k$, it follows from the minimality of $F$ that the differentials in the complex $\operatorname{Hom}_{A}(F, k)$ are trivial. Consequently, we see that $\beta_{n}(M)$ equals $\operatorname{dim}_{k} \operatorname{Ext}_{A}^{n}(M, k)$ for all $n$.

The derived category $D(A)$ is triangulated, the suspension functor $\Sigma$ being the left shift of a complex together with a sign change in the differential. Given complexes $M$ and $N$ as above, for each $n$ we may identify the cohomology group $\operatorname{Ext}_{A}^{n}(M, N)$ with $\operatorname{Hom}_{D(A)}\left(M, \Sigma^{n} N\right)$. For complexes $X$ and $Y$ in $D(A)$, denote the graded $A$ module $\oplus_{n=0}^{\infty} \operatorname{Ext}_{A}^{n}(X, Y)$ by $\operatorname{Ext}_{A}^{*}(X, Y)$. Using composition of maps in $D(A)$, the graded $A$-module $\operatorname{Ext}_{A}^{*}(M, M)$ becomes a ring, and $\operatorname{Ext}_{A}^{*}(M, N)$ becomes a graded right $\operatorname{Ext}_{A}^{*}(M, M)$-module. 
We end this section by recalling the notion of thickenings in an arbitrary triangulated category $\mathcal{T}$ with a suspension functor $\Sigma$ (cf. [11]). A subcategory of $\mathcal{T}$ is thick if it is a full triangulated subcategory closed under direct summands. Now let $\mathcal{C}$ and $\mathcal{D}$ be subcategories of $\mathcal{T}$. We denote by $\operatorname{thick}_{\mathcal{T}}^{1}(\mathcal{C})$ the full subcategory of $\mathcal{T}$ consisting of all the direct summands of finite direct sums of shifts of objects in $\mathcal{C}$. Furthermore, we denote by $\mathcal{C} * \mathcal{D}$ the full subcategory of $\mathcal{T}$ consisting of objects $M$ such that there exists a distinguished triangle

$$
C \rightarrow M \rightarrow D \rightarrow \Sigma C
$$

in $\mathcal{T}$, with $C \in \mathcal{C}$ and $D \in \mathcal{D}$. Now, for each $n \geqslant 2$, define inductively thick ${ }_{\mathcal{T}}^{n}(\mathcal{C})$ to be $\operatorname{thick}_{\mathcal{T}}^{1}\left(\operatorname{thick}_{\mathcal{T}}^{n-1}(\mathcal{C}) * \operatorname{thick}_{\mathcal{T}}^{1}(\mathcal{C})\right)$, and denote $\bigcup_{n=1}^{\infty} \operatorname{thick}_{\mathcal{T}}^{n}(\mathcal{C})$ by $\operatorname{thick}_{\mathcal{T}}(\mathcal{C})$. This is the smallest thick subcategory of $\mathcal{T}$ containing $\mathcal{C}$. For example, if $A$ is any ring, then thick ${ }_{D(A)}(A)$ is the thick subcategory of $D(A)$ formed by the perfect complexes.

\section{Complexes of finite CI-dimension}

Let $A$ be a local ring. Recall that a quasi-deformation of $A$ is a diagram $A \rightarrow$ $R \leftarrow Q$ of local homomorphisms, in which $A \rightarrow R$ is faithfully flat, and $R \leftarrow Q$ is surjective with kernel generated by a regular sequence. A homologically finite complex $M \in D(A)$ has finite complete intersection dimension if there exists such a quasideformation for which the complex $R \otimes_{A} M$ is perfect over $Q$. From now on, we write "CI-dimension" instead of "complete intersection dimension".

The notion of CI-dimension was first introduced for modules in [3], and adopted to complexes in $[\mathbf{1 2}]$. The terminology reflects the fact that a local ring is a complete intersection precisely when all its finitely generated modules have finite CI-dimension (cf [3, Theorem 1.3]). The same holds if we replace "modules" with "homologically finite complexes".

In order to prove the main result, we need the following lemma. It shows that finite CI-dimension is preserved in thick subcategories.

Lemma 3.1. Let $A$ be a local ring, and let $M \in D(A)$ be a homologically finite complex of finite CI-dimension. Then every complex in $\operatorname{thick}_{D(A)}(M)$ has finite CIdimension.

Proof. Let $A \rightarrow R \nleftarrow Q$ be a quasi-deformation of $A$ such that the complex $R \otimes_{A} M$ is perfect over $Q$. We show by induction that for all $n \geqslant 1$, every complex $X \in \operatorname{thick}_{D(A)}^{n}(M)$ satisfies $\operatorname{pd}_{Q}\left(R \otimes_{A} X\right)<\infty$. If $X$ is a direct summand of finite direct sums of shifts of $M$, then this clearly holds. Therefore $\operatorname{pd}_{Q}\left(R \otimes_{A} X\right)$ is finite for all complexes $X \in \operatorname{thick}_{D(A)}^{1}(M)$. Next, suppose the claim holds for all $1, \ldots, n$, and let $X$ be a complex in $\operatorname{thick}_{D(A)}^{n}(M) * \operatorname{thick}_{D(A)}^{1}(M)$. Then there exists a triangle

$$
Y \rightarrow X \rightarrow Z \rightarrow \Sigma Y
$$

in $D(A)$, where $Y$ and $Z$ are complexes in $\operatorname{thick}_{D(A)}^{n}(M)$ and $\operatorname{thick}_{D(A)}^{1}(M)$, respec- 
tively. By induction, $\operatorname{pd}_{Q}\left(R \otimes_{A} Y\right)$ and $\operatorname{pd}_{Q}\left(R \otimes_{A} Z\right)$ are both finite, hence so is $\operatorname{pd}_{Q}\left(R \otimes_{A} X\right)$. Since

$$
\operatorname{thick}_{D(A)}^{n+1}(M)=\operatorname{thick}_{D(A)}^{1}\left(\operatorname{thick}_{D(A)}^{n}(M) * \operatorname{thick}_{D(A)}^{1}(M)\right),
$$

the proof is complete.

Next, we prove the main result: if $M$ is a complex of finite CI-dimension and complexity $c$, then every $0 \leqslant t \leqslant c$ "occurs" as a complexity in the thick subcategory generated by $M$.

Theorem 3.2. Let $A$ be a local ring, and let $M \in D(A)$ be a nonzero homologically finite complex of finite CI-dimension. Then for every $0 \leqslant t \leqslant \mathrm{cx} M$, there exists a nonzero complex in thick $_{D(A)}(M)$ of complexity $t$.

Proof. If $c=0$, then there is nothing to prove, so suppose that $c$ is nonzero. By Lemma 3.1, it suffices to construct a nonzero complex in $\operatorname{thick}_{D(A)}(M)$ of complexity $c-1$.

Let $Z_{A}(M)$ be the graded $A$-subalgebra of $\operatorname{Ext}_{A}^{*}(M, M)$ generated by the central elements in degree two. We first show that the $Z_{A}(M)$-module $\operatorname{Ext}_{A}^{*}(M, k)$ is Noetherian, by adopting the arguments from the proof of [3, Theorem 4.9]. Let $A \rightarrow R \nleftarrow Q$ be a quasi-deformation of $A$ such that $\operatorname{pd}_{Q}\left(R \otimes_{A} M\right)$ is finite, and let $Z_{R}\left(R \otimes_{A} M\right)$ be the graded $R$-subalgebra of $\operatorname{Ext}_{R}^{*}\left(R \otimes_{A} M, R \otimes_{A} M\right)$ generated by the central elements in degree two. By [5, Corollary 5.1], the $Z_{R}\left(R \otimes_{A} M\right)$-module $\operatorname{Ext}_{R}^{*}\left(R \otimes_{A} M, R \otimes_{A} k\right)$ is Noetherian, where $k$ is the residue field of $A$. Moreover, by [3, Theorem 4.9], we may identify $Z_{R}\left(R \otimes_{A} M\right)$ with $R \otimes_{A} Z_{A}(M)$, and so, by faithfully flat descent, the $Z_{A}(M)$-module $\operatorname{Ext}_{A}^{*}(M, k)$ is Noetherian (see also [4, $\left.\S 7\right]$ ).

By [7, Lemma 2.5], there exists a positive degree element $\eta \in Z_{A}(M)$ with the property that scalar multiplication

$$
\operatorname{Ext}_{A}^{n}(M, k) \stackrel{\cdot \eta}{\longrightarrow} \operatorname{Ext}_{A}^{n+|\eta|}(M, k)
$$

is injective for $n \gg 0$. This element corresponds to a map $M \stackrel{f_{\eta}}{\longrightarrow} \Sigma^{|\eta|} M$ in $D(A)$, and completing this map we obtain a triangle

$$
M \stackrel{f_{\eta}}{\longrightarrow} \Sigma^{|\eta|} M \rightarrow K \rightarrow \Sigma M
$$

in thick ${ }_{D(A)}(M)$. Note that the object $K$ is nonzero; if not, then $M$ would be isomorphic to $\Sigma^{|\eta|} M$, and this is impossible since $|\eta| \geqslant 1$ and the nonzero complex $M$ is homologically finite. The triangle induces a long exact sequence

$$
\cdots \rightarrow \operatorname{Ext}_{A}^{n}(K, k) \rightarrow \operatorname{Ext}_{A}^{n-|\eta|}(M, k) \stackrel{\cdot(-1)^{n} \eta}{\longrightarrow} \operatorname{Ext}_{A}^{n}(M, k) \rightarrow \operatorname{Ext}_{A}^{n+1}(K, k) \rightarrow \cdots
$$

in cohomology; hence for some integer $n_{0}$ the equality $\beta_{n+1}(K)=\beta_{n}(M)-\beta_{n-|\eta|}(M)$ 
holds for $n \geqslant n_{0}$. The Poincaré series of $K$ is then given by

$$
\begin{aligned}
P(K, t) & =\sum_{n=0}^{\infty} \beta_{n}(K) t^{n} \\
& =\sum_{n=0}^{n_{0}} \beta_{n}(K) t^{n}+\sum_{n=n_{0}+1}^{\infty} \beta_{n}(K) t^{n} \\
& =\sum_{n=0}^{n_{0}} \beta_{n}(K) t^{n}+\sum_{n=n_{0}+1}^{\infty}\left(\beta_{n-1}(M)-\beta_{n-|\eta|-1}(M)\right) t^{n} \\
& =g(t)+\left(t-t^{|\eta|+1}\right) P(M, t) \\
& =g(t)+t\left(1+t+\cdots+t^{|\eta|-1}\right)(1-t) P(M, t),
\end{aligned}
$$

where $g(t)$ is a polynomial in $\mathbb{Z}[t]$.

By [12, Corollary 3.10(iv)], the Poincaré series $P(M, t)$ of $M$ is rational, hence so is $P(K, t)$. Therefore, the complexities of $M$ and $K$ equal the orders of the poles at $t=1$ of $P(M, t)$ and $P(K, t)$, respectively. Consequently, the complexity of $K$ is cx $M-1$. Moreover, we see from the construction of $K$ that this complex belongs to thick $_{D(A)}(M)$. This completes the proof.

The main idea in the previous proof is also present in the proof of $[6$, Proposition 6.1], namely, the use of iterated mapping cones to build complexes of prescribed complexity. In the above proof, the central elements used to form these mapping cones belong to the centers of the Ext-algebras of the complexes involved. However, when the ring is a complete intersection, these central elements come from the center of the derived category, as in $[\mathbf{6}]$.

Using $[8,3.8]$, the following corollary follows immediately from Theorem 3.2. It settles the question raised in [8, Remarks 9.6]: every nonzero homologically finite

complex of finite CI-dimension over a local ring is virtually small. In other words, the thick subcategory is generates contains a nonzero perfect complex.

Corollary 3.3. Let $A$ be a local ring, and let $M \in D(A)$ be a nonzero homologically finite complex of finite CI-dimension. Then

$$
\operatorname{thick}_{D(A)}(M) \cap \operatorname{thick}_{D(A)}(A)
$$

is nonzero.

\section{References}

[1] L. Avramov, Modules of finite virtual projective dimension, Invent. Math. 96 (1989), no. 1, 71-101.

[2] L. Avramov, Homological asymptotics of modules over local rings, in Commutative algebra (Berkeley, 1987) (M. Hochster, C. Huneke, J. Sally, eds.), MSRI Publ. 15, Springer-Verlag, New York 1989, pp. 33-62.

[3] L. Avramov, V. Gasharov, and I. Peeva, Complete intersection dimension, Inst. Hautes Études Sci. Publ. Math. 86 (1997), 67-114 (1998). 
[4] L. Avramov and S.B. Iyengar, Constructing modules with prescribed cohomological support, Illinois J. Math. 51 (2007), no. 1, 1-20.

[5] L. Avramov and L.-C. Sun, Cohomology operators defined by a deformation, J. Algebra 204 (1998), no. 2, 684-710.

[6] D.J. Benson and J.P.C. Greenlees, Complete intersections and derived categories, preprint, 2009.

[7] P.A. Bergh, S.B. Iyengar, H. Krause, and S. Oppermann, Dimensions of triangulated categories via Koszul objects, Math. Z., in press.

[8] W. Dwyer, J.P.C. Greenlees, and S. Iyengar, Finiteness in derived categories of local rings, Comment. Math. Helv. 81 (2006), no. 2, 383-432.

[9] T.H. Gulliksen, On the deviations of a local ring, Math. Scand. 47 (1980), no. 1, $5-20$.

[10] P. Roberts, Homological invariants of modules over commutative rings, Séminaire de Mathématiques Supérieures [Seminar on Higher Mathematics] 72, Presses de l'Université de Montréal, Montreal, Que., 1980.

[11] R. Rouquier, Dimensions of triangulated categories, J. K-Theory 1 (2008), no. 2, 193-256.

[12] S. Sather-Wagstaff, Complete intersection dimensions for complexes, J. Pure Appl. Algebra 190 (2004), no. 1-3, 267-290.

Petter Andreas Bergh bergh@math.ntnu.no

Institutt for matematiske fag, NTNU, N-7491 Trondheim, Norway 